\title{
Role of disorder on transport in boron-doped multiwalled carbon nanotubes
}

\author{
V. Krstić, ${ }^{1, *}$ S. Blumentritt, ${ }^{1}$ J. Muster, ${ }^{1}$ S. Roth, ${ }^{1}$, and A. Rubio, ${ }^{2,3}$ \\ ${ }^{1}$ Max-Planck-Institut für Festkörperforschung, Heisenbergstraße 1, D-70569 Stuttgart, Germany \\ ${ }^{2}$ Departamento de Fisica de Materiales, Facultad de Quimicas, Universidad del Pais Vasco/Euskal Herriko Unibertsitatea UPV/EHU \\ and Centro Mixto CSIC-UPV/EHU, Spain \\ ${ }^{3}$ Donostia International Physics Center (DIPC), Apdo. 1072, 20018 San Sebastian/Donostia, Spain
}

(Received 22 July 2002; published 13 January 2003)

\begin{abstract}
Electrical transport measurements on boron-doped multiwalled carbon nanotubes deposited on top of predefined electrode patterns have been performed. The temperature dependence of the conductance reveals through two-point configuration, the appearance of a zero-bias anomaly which was found to be not compatible with the existence of a Luttinger-liquid-like state in the doped multiwalled carbon nanotubes. The experimental findings indicate that, in order to properly interpret the charge transport properties of multiwalled carbon nanotubes, the doping as well as the energy window in which the experiments are performed are crucial points.

DOI: 10.1103/PhysRevB.67.041401

PACS number(s): 73.63.Fg, 72.80.Rj, 73.22.Lp
\end{abstract}

Multiwalled carbon nanotubes (MWNTs), in contrast to single-walled carbon nanotubes (SWNTs), consist of a number of stacked-in shells and have a diameter typically of tens of nm's which is one order of magnitude larger than for SWNTs. Despite these differences, MWNTs are often regarded as one-dimensional (1D) systems, with respect to their electronic properties in the same manner as SWNTs. SWNTs are expected to behave as quasi 1D molecular wires with large mean free paths [typically $1 \mu \mathrm{m}$ (Ref. 1)], so that ballistic charge transport is observable. ${ }^{2-6}$ As a direct consequence of the 1D-conducting behavior of carbon nanotubes, strong Coulomb interaction effects are expected that imply a breakdown of the Fermi-liquid theory leading to a Luttingerliquid response characterized by a pronounced power-law suppression of the transport current and density of states. ${ }^{7}$ The situation for MWNTs is more complex. Earlier experiments ${ }^{8}$ found evidence of electron scattering and the transport appeared diffusive rather than ballistic. However, experiments done by dipping a MWNT in a liquid metal ${ }^{5}$ show ballistic transport and the ability to carry large electrical currents. These observations suggest that the electrons are well decoupled from the underlying lattice, that is, only a weak electron-phonon interaction is apparent, similar to SWNTs. ${ }^{9}$ At low temperatures several contradictory observations in the electronic transport properties of MWNTs have been made: magnetotransport data could be successfully interpreted by the Altshuler-Aronov-Spivak effect ${ }^{10}$ and in the framework of weak-localization theory, ${ }^{11}$ which both point toward a disordered electronic state with 2D character at low temperatures. On the other hand a zero-bias anomaly (ZBA) in the conductance has been reported. ${ }^{12}$ This could be interpreted by the existence of a Luttinger-liquid (LL)-like state which requires a $1 \mathrm{D}$ electronic system with repulsive interaction between the electrons.

In the present work we report on temperature-dependent electronic transport measurements on boron (B) -doped MWNTs (B-MWNTs) with approximately $1 \%$ B content which were produced by the arc-discharge method. The use of B-MWNTs aims toward the investigation of the intershellcoupling between neighboring shells, dimensionality of the electron system and the degree of disorder introduced by the dopant. Based on the experimental findings, recalling that B doping implies the creation of lattice defects, a possible explanation is proposed for the above discrepancies in electronic transport in undoped MWNTs. The samples were prepared as follows: first the B-MWNT raw material was dispersed in surfactant solution and then purified via centrifugation following the lines of Ref. 13. After purification, tubes from the dispersion were adsorbed on top of electronbeam lithographically predefined AuPd patterns. As substrate, a $\mathrm{Si}$ wafer with a thermally grown $\mathrm{SiO}_{2}$ layer on top was used. Before adsorption, the $\mathrm{SiO}_{2}$ surface and the electrode lines were illuminated $(30 \mathrm{~min})$ with UV light, and afterward thoroughly rinsed with aceton and isopropanol in order to eliminate contaminations, especially from the metal electrodes. ${ }^{13}$

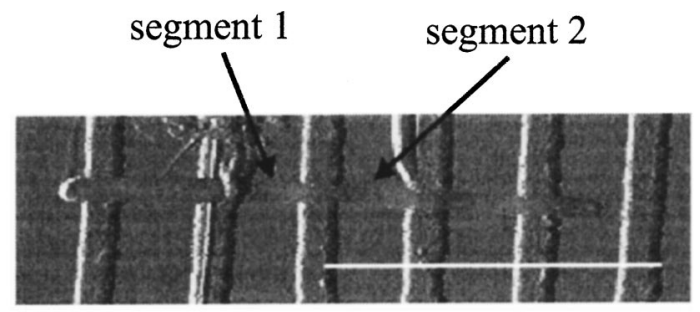

(a)

\begin{tabular}{|c|c|c|c|}
\hline & $R_{4 T}[\mathrm{k} \Omega]$ & $G_{\exp }\left[\mathrm{m} \Omega^{-1}\right]$ & $L[\mathrm{~nm}]$ \\
\hline segment 1 & 5.0 & 0.2 & 200 \\
\hline segment 2 & 5.7 & 0.175 & 220 \\
\hline segment 1+2 & 13.0 & 0.077 & 500 \\
\hline
\end{tabular}

(b)

FIG. 1. (a) Image of a B-MWNT on top of AuPd electrodes taken by scanning force microscopy. The electrode fingers are about $125 \mathrm{~nm}$ in width, $14 \mathrm{~nm}$ in height, and separated by approximately $240 \mathrm{~nm}$. The height of the B-MWNT was found to be about $7 \mathrm{~nm}$ determined by SFM measurements. The white bar corresponds to 1 $\mu \mathrm{m}$. Segments 1 and 2 are indicated. (b) Table summarizing the segment lengths $L$, the four-terminal resistance $R_{4 T}$ and thus also the conductance $G_{\text {exp }}=1 / R_{4 T}$ of each segment as well as their sum at room temperature. Apparently, the four-terminal resistance $R_{4 T}$ and thus also $G_{\exp }$ change significantly with length $L$ indicating intershell-interactions in the B-MWNT. 

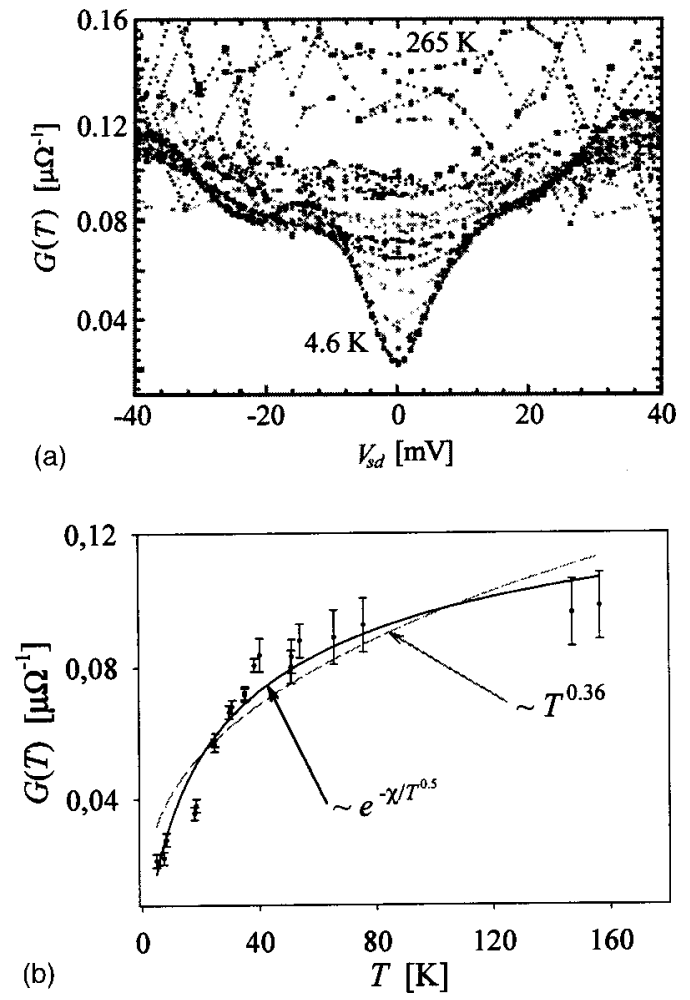

FIG. 2. (a) Differential conductance $G$ of a B-MWNT as a function of applied source-drain-voltage $V_{\text {sd }}$ in two-terminal configuration. The temperature was varied from 265 to $4.6 \mathrm{~K}$. A wellpronounced ZBA around zero $V_{\text {sd }}$ is observed. (b) $T$ dependence of the ZBA. Obviously, the fit assuming $G(T) \sim e^{-\chi / \sqrt{T}}$ and $\chi=4.86$ $\pm 0.38 \mathrm{~K}^{0.5}$, coincides much better with the experimental data than the fit $G(T) \sim T^{0.36}$.

In Fig. 1(a) a scanning force microscopy (SFM) image of a typical sample is shown. The deposited tube has an outer diameter of about $7 \mathrm{~nm}$ such that the number of shells can be estimated to be between 8 and 12. In Fig. 1(b) a table is given providing the room-temperature four-terminal resistance $R_{4 T}$ and conductance the $G_{\text {exp }}=1 / R_{4 T}$ of the corresponding B-MWNT segments 1 and 2 . These values are comparable to the data in Ref. 14.

For MWNTs it is known that the intershell coupling has to be taken into account when analyzing the experimental electrical transport data as demonstrated in Refs. 5 and 15. Furthermore, according to Ref. 16 the conductance of a (metallic) MWNT with intershell coupling also depends on whether neighboring shells are commensurate or incommensurate. Based on these two points, the conductance of a MWNT has a power-law dependence on its length $L$ given by

$$
G(L) \sim L^{(\eta-1) / \eta}
$$

where the exponent $\eta$ is a measure for the MWNT whether it has to be regarded as a disordered or ballistic conductor. The case $\eta=\frac{1}{2}$ accounts for a disordered system whereas $\eta=1$ points toward a ballistic charge transport, i.e., $G(L)$ =const. ${ }^{16}$ Furthermore, the case $\eta=1$ implies that the MWNT consists of commensurate shells. ${ }^{16}$ Fitting the data on $G_{\exp }$ given in the table in Fig. 1(b) with the aid of Eq. (1)

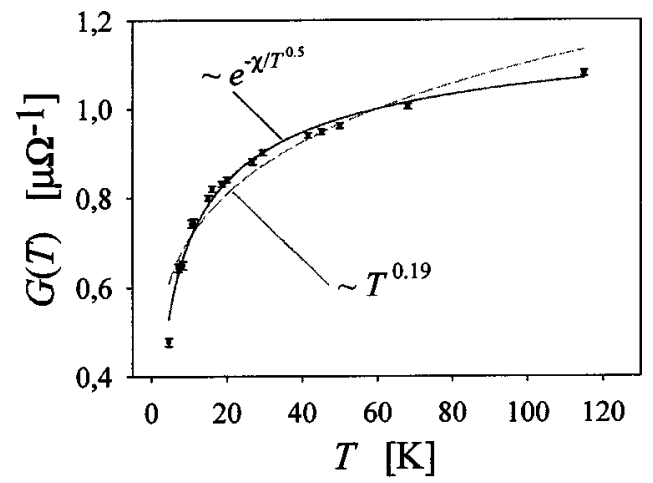

FIG. 3. T-dependence of the ZBA for another B-MWNT sample. Again the fit assuming a disordered conducting wire, $G(T)$ $\sim T^{-\chi / \sqrt{T}}$ and $\chi=1.88 \pm 0.07 \mathrm{~K}^{0.5}$, describes the experimental data considerably better than the fit which assumes the B-MWNT to be in a LL-like state, $G(T) \sim T^{0.19}$.

yields $\eta_{\mathrm{B}-\mathrm{MWNT}} \approx 0.49 \pm 0.02$. This value indicates that the B-MWNTs have scattering centers that originate from structural or topological defects and the likely geometrical incommensurability of neighboring shells ${ }^{16}$ such that diffusive charge transport is apparent. In consequence, the B-MWNT is not expected to show strong LL-like features; instead a more 2D (i.e., Fermi-liquid-like) character of the electron system should be apparent. The 2D character is also supported by the circumstance that the LL state in a MWNT approaches the Fermi-liquid state for increasing number of shells. ${ }^{17}$

In Fig. 2(a) the differential conductance vs applied source-drain voltage $V_{\text {sd }}$ is shown for a B-MWNT for different temperatures. A well-pronounced ZBA is observed, which increases with decreasing temperature. In Fig. 2(b) the temperature $(T)$ dependence of the conductance minimum at zero $V_{\mathrm{sd}}$ is plotted. The dashed curve corresponds to a fit for which the B-MWNT was assumed to be in a LL-like state, i.e., $G(T) \sim T^{\alpha}$. For the exponent $\alpha$ the approximate value 0.36 was found. This value is of similar magnitude to the one observed in Ref. 12. Apparently the LL fit and the experimental data do not coincide very well. In contrast, a fit (continuous gray line) according to

$$
G(T) \sim e^{-\chi / \sqrt{T}}
$$

accounts considerably better for the experimental data. The fit is based on a recent theoretical work by Mishchenko et al., ${ }^{18}$ who described a metallic MWNT as a disordered wire. Within this theoretical framework the ZBA originates from the impeded redistribution of charges in the MWNT due to the finite propagation time of charge carrying plasmon. That is, the MWNT is longer than the plasmon wavelength, and thus scattering processes of the plasmon gain relevance. ${ }^{18}$ The parameter $\chi$ is a measure of the degree of disorder in the MWNT and was found to be 4.86 $\pm 0.38 \mathrm{~K}^{0.5}$ from the fit to the data in Fig. 2(b). The value of $\chi$ depends on the number of shells of the MWNT under investigation, the interaction strength between the electrons and the momentum relaxation time of the charge carriers. ${ }^{18}$ In Fig. 3 the $T$ dependence of the ZBA of another B-MWNT 
is shown. Similar to the data in Fig. 2, the LL fit (dashed line) does not coincide as good as the fit (continuous gray line) following Eq. (2) for which $\chi=1.88 \pm 0.07 \mathrm{~K}^{0.5}$ is found. Comparing the parameter $\chi$ extracted for the two MWNT samples, the first investigated MWNT seems to have a higher degree of disorder than the second MWNT, which indicates a possible variation of the B dopant concentration for the two tubes.

For the LL fit in Fig. 3 the exponent $\alpha$ was found to be approximately 0.19 . However, this value would imply a considerably weaker electron-electron-interaction for this B-doped MWNT sample than in the previously presented sample, which is unlikely.

The data presented in Fig. 2 and 3 point toward a fully disordered electronic system rather than a LL state in the B-MWNTs investigated. Noteworthy at this point is that works on large-diameter B-MWNTs (Ref. 14) report on deviations from the LL picture, too, but they still could fit the conductance by a $T^{\alpha}$ dependence.

Our experimental data indicate that B-MWNTs are 2D diffusive metallic conductors, in contrast to undoped MWNTs which are believed to be 1D ballistic nanowires. That is, doping destroys the 1D character of the undoped MWNT. As the content of $\mathrm{B}$ atoms in the investigated B-MWNTs is rather low, the analysis presented here also provides insight in the discrepancy in experimental results for undoped MWNTs. Since B atoms in B-MWNTs are lattice defects, the electronic properties of the B-MWNT will depend on the density of these defects. In view of undoped MWNTs, similarly, the density of defects (e.g., pentagonheptagon defects), vacancies or chemical side groups as well as the commensurability of neighboring shells is likely to influence the charge transport along the tube. In order to follow this it has to be taken into account that the usual lattice defects only weakly modify the charge transport along an undoped MWNT. ${ }^{19,20}$ A similar argument has been used to explain that disorder introduced in a metallic tube affects the electrical transport properties much less than in a semiconducting tube ${ }^{21}$ It has to be noted that in the case of a MWNT only one third of the shells can be expected to be metallic. Thus the majority of shells in a MWNT are usually semiconducting, and therefore more susceptible to the influence of disorder. Regarding previous works on B-MWNTs, ${ }^{14}$ one may also speculate that altering of the B defect/electron scat- tering cross-section due to a reduced curvature allows for the creation of an intermediate state between LL and a fully disordered system.

It can be concluded that, even for a high density of defects, effects like ballistic transport ${ }^{5}$ may be observable at high temperatures, and the MWNT could still obey a 1D electronic character. However, increasing the density of defects is likely to lead to a shift towards 2D-like diffusive electronic transport, which can then show weak localization ${ }^{11}$ and the Altshuler-Aronov-Spivak effect ${ }^{10}$ at low temperatures. In case the density of defects is relatively low, a LL-like electronic state can form showing signatures like a ZBA. ${ }^{12}$ It is noteworthy at this point that the theoretical considerations in Ref. 18 allow for the case that even though at a certain defect density and temperature regime LL-like behavior may be found in undoped MWNTs, at lower temperatures a crossover to a disordered 2D system is possible. As a consequence, from the experimental point of view the energy window, i.e., temperature range and applied source-drain voltage, within which the experiments are performed, plays a crucial role for the observation of certain electrical transport phenomena in undoped MWNTs and B-MWNTs.

Summarizing, the data and analysis presented here indicate that B-MWNTs are, at high temperatures, 2D-like diffusive metallic conductors with intershell coupling. At low temperatures a ZBA is observed similar to a LL system. However, the ZBA could only be reasonably fitted under the assumption that the B-MWNT is a disordered conducting wire, ${ }^{18}$ but not in a LL-like electronic state. Analogous considerations regarding defect density in undoped MWNTs with reported experiments may provide a possible explanation of the discrepancy in observed electrical transport phenomena. ${ }^{10-12}$

\section{ACKNOWLEDGMENTS}

The authors thank Ph. Kohler-Redlich and F. Zha for providing B-doped MWNT raw material and S. Roche for discussions about the transport in incommensurate structures. We acknowledge financial support from the Basque Gouvernement, Spanish DGES, and the European RTN network Contract Nos. HPRN-CT-2000-00128 (COMELCAN) and RTD-FET program (SATUNET).
*Corresponding author. Email address: v.krstic@fkf.mpg.de Fax: 0049-(0)711-689 1010.

${ }^{1}$ C. T. White and T. N. Todorov, Nature (London) 393, 240 (1998).

${ }^{2}$ S. J. Tans, M. H. Devoret, H. Dai, A. Thess, R. E. Smalley, L. J. Geerligs, and C. Dekker, Nature (London) 386, 474 (1997); S. J. Tans, M. H. Devoret, R. J. A. Groeneveld, and C. Dekker, ibid. 394, 761 (1998).

${ }^{3}$ M. Bockrath, D. Cobden, P. McEuen, N. G. Chopra, A. Zettl, A. Thess, and R. E. Smalley, Science 275, 1922 (1997); D. H. Cobden, M. Bockrath, P. L. McEuen, A. G. Rinzler, and R. Smalley, Phys. Rev. Lett. 81, 681 (1998).

${ }^{4}$ A. Bachtold, M. Fuhrer, S. Plyasunov, M. Forero, E. H. Anderson,
A. Zettl, and P. L. McEuen, Phys. Rev. Lett. 84, 6082 (2000).

${ }^{5}$ S. Frank, P. Poncharal, Z. L. Wang, and W. A. de Heer, Science 280, 1744 (1998).

${ }^{6}$ V. Krstić, S. Roth, and M. Burghard, Phys. Rev. B 62, R16 353 (2000).

${ }^{7}$ M. Bockrath, D. Cobden, J. Liu, A. G. Rinzler, R. Smalley, L. Balents, and P. L. McEuen, Nature (London) 397, 598 (1999). For a review of Luttinger liquid results in carbon nanotubes, see R. Egger, A. Bachtold, M. Fuhrer, M. Bockrath, D. Cobden, and P. L. McEuen, in Interacting Electrons in Nanostructures, edited by R. Haug and H. Schoelle (Springer, Berlin, 2002), and references therein.

${ }^{8}$ L. Langer, V. Bayot, E. Grivei, J. P. Issi, J. P. Heremanns, C. H. 
Olk, L. Storkmann, C. Van Haesendonck, and Y. Bruynseraede, Phys. Rev. Lett. 76, 479 (1996).

${ }^{9}$ T. Hertel and G. Moss, Phys. Rev. Lett. 84, 5002 (2000).

${ }^{10}$ A. Bachtold, C. Strunk, J.-P. Salvetat, J.-M. Bonard, L. Forró, T. Nussbaumer, and C. Schönenberger, Nature (London) 397, 673 (1999).

${ }^{11}$ A. Fujiwara et al., in Molecular Nanostructures, edited by $\mathrm{H}$. Kuzmany, J. Fink, M. Mehring, and S. Roth (World Scientific, Singapore, 1998), pp. 439-442.

${ }^{12}$ C. Schönenberger, A. Bachtold, C. Strunk, J.-P. Salvetat, and L. Forró, Appl. Phys. A: Mater. Sci. Process. 69, 283 (1999); A. Bachtold, M. de Jonge, K. Grove-Rasmussen, P. L. McEuen, M. Buitelaar, and C. Schönenberger, Phys. Rev. Lett. 87, 166801 (2001).

${ }^{13}$ V. Krstić, J. Muster, G. S. Duesberg, G. Philipp, M. Burghard, and S. Roth, Synth. Met. 110, 245 (2000).

${ }^{14}$ K. Liu, Ph. Avouris, R. Martel, and W. K. Hsu, Phys. Rev. B 63,
161404 (2001).

${ }^{15}$ S. Sanvito, Y.-K. Kwon, D. Tomanek, and C. J. Lambert, Phys. Rev. Lett. 84, 1974 (2000).

${ }^{16}$ S. Roche, F. Triozon, A. Rubio, and D. Mayou, Phys. Lett. A 285, 94 (2001).

${ }^{17}$ R. Egger, Phys. Rev. Lett. 26, 5547 (1999).

${ }^{18}$ E. G. Mishchenko, A. V. Andreev, and L. I. Glazman, Phys. Rev. Lett. 87, 246801 (2001); E. G. Mishchenko and A. V. Andreev, Phys. Rev. B 65, 235310 (2002).

${ }^{19}$ A. Bachtold, M. Henny, C. Terrer, C. Strunk, C. Schönenberger, J.-P. Salvetat, J.-M. Bonard, and L. Forró, Appl. Phys. Lett. 73, 274 (1998).

${ }^{20}$ F. Beuneu, C. l'Huillier, J.-P. Salvetat, J.-M. Bonard, and L. Forró, Phys. Rev. B 59, 5945 (1999).

${ }^{21}$ P. L. McEuen, Marc Bockrath, David H. Cobden, Young-Gui Yoon, and Steven G. Louie, Phys. Rev. Lett. 83, 5098 (1999). 